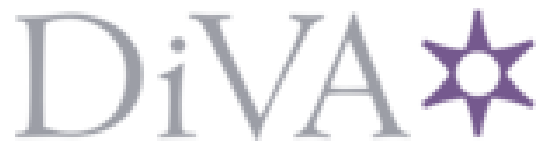

http://www.diva-portal.org

This is the published version of a paper published in Journal of addiction medicine.

Citation for the original published paper (version of record):

Jemberie, W B., Padyab, M., Snellman, F., Lundgren, L. (2020)

A Multidimensional Latent Class Analysis of Harmful Alcohol Use Among Older Adults:

Subtypes Within the Swedish Addiction Severity Index Registry

Journal of addiction medicine, 14(4): e89-e99

https://doi.org/10.1097/ADM.0000000000000636

Access to the published version may require subscription.

N.B. When citing this work, cite the original published paper.

Permanent link to this version:

http://urn.kb.se/resolve?urn=urn:nbn:se:umu:diva-168621 


\title{
A Multidimensional Latent Class Analysis of Harmful Alcohol Use Among Older Adults: Subtypes Within the Swedish Addiction Severity Index Registry
}

\author{
Wossenseged Birhane Jemberie, MSc, Mojgan Padyab, PhD, \\ Fredrik Snellman, PhD, and Lena Lundgren, PhD
}

\begin{abstract}
Objectives: The present study aimed to identify multidimensional typologies of harmful alcohol use based on the Swedish Addiction Severity Index (ASI) assessment data on individuals aged 50 years and above.

Methods: Latent class analysis examined 11 indicators from ASI data on 1747 individuals $(\operatorname{men}=1255$, women $=492)$ who reported they were troubled by alcohol problem at least one day in the past 30 days before their assessment. The discriminative validity of the classes was assessed by comparing other measures of individual characteristics and problem severity of other ASI dimensions.

Results: Five subtypes of harmful alcohol use were identified. Two classes with alcohol problems varying in psychosocial functioning, age composition and ages of onset of both regular and heavy drinking. Two with psychiatric comorbidity but varying in violence, criminality, gender composition and ages of onset of regular and heavy drinking. One with high prevalence of concurrent use of other substances, psychiatric, legal, and employment problems.
\end{abstract}

From the Department of Social Work, Umeå University, Umeå, Sweden, Centre for Demography and Ageing Research (CEDAR), Umeå University, Umeå, Sweden (WBJ, MP); Department of Social Work, Umeå University, Umeå, Sweden (FS); The Cross-National Behavioral Health Laboratory, University of Denver, Denver, Colorado, Professor, Graduate School of Social Work, University of Denver, Denver, Colorado, Professor, Department of Social Work, Umeå University, Umeå, Sweden (LL). Received for publication May 2, 2019; accepted January 20, 2020.

This study was funded by grants from The Swedish Research Council for Health, Working Life and Welfare (Grants no. 2016-07213). The first author (WBJ) was also awarded grant from the Kempe Foundation to cover tuition fees for methodological courses relevant to this study.

The authors report no conflicts of interest.

Supplemental digital content is available for this article. Direct URL citation appears in the printed text and is provided in the HTML and PDF versions of this article on the journal's Web site (www.journaladdictionmedicine.com). Send correspondence to Wossenseged Birhane Jemberie, MSc, Postgraduate Student, Department of Social Work, Samhällsvetarhuset, Umeå University, 90187 Umeå, Sweden. E-mail: wossenseged.jemberie@umu.se.

Copyright $\odot 2020$ The Author(s). Published by Wolters Kluwer Health, Inc. on behalf of the American Society of Addiction Medicine. This is an open access article distributed under the terms of the Creative Commons Attribution-Non Commercial-No Derivatives License 4.0 (CCBY-NC$\mathrm{ND}$ ), where it is permissible to download and share the work provided it is properly cited. The work cannot be changed in any way or used commercially without permission from the journal.

ISSN: 1932-0620/20/1404-0e89

DOI: 10.1097/ADM.0000000000000636
Conclusions: The analysis identified, in a national sample, heterogeneous risk groups of older adults with harmful alcohol use. These findings suggest a need for healthcare providers to assess older adults not only for their substance use but also for associated problems and needs. Given these findings, the Addiction Severity Index is a valuable assessment tool for older adults with harmful alcohol use.

Key Words: addiction severity Index, harmful alcohol use, older adults, register-based study, subtypes

( $J$ Addict Med 2020;14: e89-e99)

$T$

he global population, especially in high income countries, is aging. Sweden's population is estimated to grow to 11.2 million by 2030 from 10.1 million in 2017 (an increase of $11 \%$ ) and the total number of adults aged 65 years and older is expected to increase from 2 million to 2.4 million-a $20 \%$ increase (Statistics Sweden, 2018). One major influence is the entry of "baby boomers" (children born between mid-1940s and mid 1960s) to late adulthood.

Although an early death is common for individuals with substance use disorders (SUD), aging baby boomers, increased availability of treatment for alcohol and drug use disorders and medications for alcohol and opioid use disorders, changes in patterns of use, access to health care and services such as harm-reduction programs mean that, compared to earlier cohorts, individuals with SUD are living longer (Beynon, 2009; Han et al., 2009; Arndt et al., 2011; Carew and Comiskey, 2018). Given this trend, the number of older adults with substance use problems is growing (Sorocco and Ferrell, 2006; Kuerbis et al., 2014). In the US, for example, the number of adults aged 50 or older with substance use disorder is projected to increase from 2.8 million in 2006 to 5.7 million in 2020 (Han et al., 2009). Swedish data suggest a similar trend. Taking alcohol use disorder (AUD), the primary substance use problem among older adults, the proportion of at-risk alcohol drinkers 65 to 84 years of age increased from 5\% to 11\% between 2004 and 2016 (Public Health Agency of Sweden, 2016). One in four Swedish patients $(27 \%)$, moreover, hospitalized for a primary or secondary alcohol-related diagnosis in 2004 were adults aged 60 or older and increased to $40 \%$ in 2015 (Public Health Agency of Sweden, 2016). Adverse health outcomes related to use of other psychoactive substances also increased among 
older adults. Deaths related to drugs among adults aged 50 years and above increased from $26 \%$ in 2000 to $36 \%$ in 2015 (CAN, 2016).

Detection of SUD in older adults can be challenging due to atypical presentations and other existing pathologies. Agerelated physical and cognitive decline may mask symptoms of SUD and practitioners often attribute such declines and incidents such as falling and hip fractures to ageing and frailty, sometimes resulting failure to assess SUD (Flint et al., 2018). Other major barriers to identification of SUD in older adults include ageist assumptions, discomfort in practitioners asking older adults about their substance use, reluctance to refer appropriately and arbitrary age limits for treatment admission making treatment less accessible to older adults (Badrakalimuthu et al., 2010; Lichtenberg, 2010; Royal College of Psychiatrists, 2018).

\section{Previous Alcohol Typology Studies}

Clinical practitioners and researchers have developed systems and theories on the nature of alcohol problems to describe and categorize clinical profiles of individuals with problematic alcohol use. Some were intuitive in origin (Jellinek, 1960), other studies used a priori groupings based on variables or traits (eg, gender, parental alcoholism, presence of psychiatric problem) of theoretical interest. Empirical approaches, conversely, utilized $a$ posteriori statistical procedures (Babor et al., 1992a). As the conceptual understanding of alcohol use disorders and other substance use disorders evolved to focus on the problem's multidimensionality, typology studies using sophisticated statistical approaches and measured multiple indicators started to emerge in the literature (Cloninger et al., 1981; Morey et al., 1984; Babor et al., 1992b). Of these, Cloninger's "Type 1/Type 2" and Babor's "Type A/B" typologies remain the most influential. Note, when discussing previous studies in this section, we use the terminologies the authors used related to the spectrum of unhealthy substance use (in italics below).

Cloninger's "Type 1/Type 2" typology, based on analysis of Swedish adoptees and their biological and adoptive parents, had inheritance of alcoholism as a classification criterion (Bohman et al., 1981; Cloninger et al., 1981). Type 1 subtype reflected a late age of onset among men and women who had mild to severe drinking problems. The risk factors for Type 1 alcoholism were both genetic and environmental. Individuals with Type 1 alcoholism had fewer alcohol-related problems, fewer inpatient treatment admissions, and were generally cautious, avoided risky practices, and drank to relieve anxiety. The "Type 2" subtype was described with primarily genetic risk factors, male-represented, with early age of onset, moderate alcohol severity but severe alcoholrelated problems (such as criminality), and personality traits of high novelty seeking, low harm avoidance and low reward dependence.

Babor's "Type A/B" typology was derived from a study of inpatient individuals with alcoholism using 17 clustering variables assessing biopsychosocial attributes of alcoholism. The variables included premorbid risk factors (eg, familial alcoholism), severity of alcohol and other drugs use, concurrent psychiatric problems, chronicity and consequences of alcohol use. The "Type A" cluster was characterized by late onset of alcoholism, fewer childhood risk factors and familial alcoholism, less psychiatric comorbidity, and fewer alcoholrelated problems. The "Type B" cluster featured high polydrug use, severe and chronic alcohol use, early onset of alcoholism, history of repeated treatment, familial history of alcoholism and presence of psychiatric comorbidity.

Although these 2 typologies encompass the multidimensionality of alcohol use disorder, their binary model of classification is insufficient to capture the heterogeneity of alcohol use disorders. A later study, using the Babor et al. (1992b) data, for example, found two additional genderspecific clusters: one with higher proportion of women and high prevalence of depression and anxiety, and another maledominated cluster characterized with high levels of alcohol use, social consequences and antisocial personality (DelBoca and Hesselbrock, 1996). Studies using advanced statistical procedures reported up to five-type models. A more detailed description of these studies is available elsewhere (Hesselbrock and Hesselbrock, 2006; Leggio et al., 2009).

These typologies were derived from younger study populations. This means, the findings from these studies may not generalize to older adults. Older and younger adults may vary in their stressors and other biopsychosocial factors associated with alcohol use disorders. Retirement, reduced social networks, loss of loved ones and social isolation may contribute to the onset and maintenance of alcohol and drug use in older adults (Akerlind and Hornquist, 1992; Perreira and Sloan, 2001; Kuerbis and Sacco, 2012; Kim et al., 2018; Kuerbis et al., 2018). Older adults may also have longer exposure to substances (early onset) and face more severe health outcomes (Moos et al., 2009) leading to early retirement and early needs for elderly care services. The Sweden National Board of Health and Welfare (NBHW) reported that the proportion of adult clients aged 65 years and older with substance use disorders who received social services, (eg, individual, means-tested out-patient care and housing assistance) almost doubled between 2007 and 2018. Furthermore, alcohol use disorder is the primary substance use problem in this age group and the most common reason for compulsory care (The National Board of Health and Welfare, 2018).

\section{Previous Alcohol Typology Studies on Older Adults}

Few analyses of older adults (50 years and older) have examined alcohol typologies. Graham et al. (1993) employed an intuitive approach to develop an alcohol typology for older adults based on a sample of 36 individuals. Their three types included: (1) "Chronic alcohol abuser" — those with chronic and primarily habitual alcohol use, repeated treatment history and early retirement before the age of 65. (2) "Reactive problem drinker" - those with late onset of problem drinking (caused by loss, grief and ill health), but with stable housing, stable job history and social support. (3) "Problem drinkers with comorbid psychiatric or cognitive problems" (mostly women, however, onset for problem drinking, existing loss and members' reaction to stressors varied). Even if the subtypes identified in this study have theoretical support, the small sample size limits generalizability. 
The most common criterion to generate alcohol typologies a priori in older adults is age of onset (Rosin and Glatt, 1971; Wetterling et al., 2003; van den Berg et al., 2014; Schückher et al., 2017; Van Montfoort-De Rave et al., 2017). Dichotomous unidimensional classifications, however, do not consider the biopsychosocial attributes of alcohol use disorder and often fail to yield subtypes with unique characteristics. Robins and Guze (1970) recommend that a step in systematic classification should be clinical description, where the clinical picture is defined not only by the striking clinical symptoms but also by other biopsychosocial factors. Inclusion of such attributes in a typology study can enhance understanding of the characteristics of heterogeneous groups of individuals with differing problem severity and treatment indications. This improved clinical insight may translate into more effective interventions.

Given the dearth of knowledge about alcohol typologies among older adults and the proposition that no single criterion can be applied to effectively develop heterogeneous subtypes, the current study seeks to identify multidimensional typologies of harmful alcohol use among individuals aged 50 years and older. Such identification of relevant typologies may facilitate diagnosis and treatment by characterizing the nature of alcohol problems and the various clinical and demographic differences among older adult treatment seekers.

\section{METHODS}

\section{Setting and Participants}

In Sweden, assessment for SUD uses the Addiction Severity Index (ASI) instrument (McLellan et al., 1992). The ASI records participant demographic characteristics and examines seven potential problem areas (dimensions) commonly affected by unhealthy substance use: somatic health, employment and self-support, alcohol use, drug use, legal problems, family and social relationships, and psychiatric health. It also collects information on familial history of substance use and mental health status. A standardized Swedish version of the ASI has been used in Sweden since the 1990s (Nyström et al., 2009) and has good validity and reliability (Makela, 2004; Armelius et al., 2009; Nystrom et al., 2010; Lundgren and Krull, 2018). Clinical social workers, who conduct the assessments, are trained and certified as ASI-interviewers. Nearly $70 \%$ of municipalities in Sweden use the ASI to map the individual's living situation, assess severity of a substance use problem, client treatment needs and to follow-up interventions and record intervention outcomes (Lundgren et al., 2014). These data are entered into a national register created by the National Board of Health and Welfare, forming the national ASI-database.

Between 2003 and 2017, 15,061 unique individuals aged 18 years and older, from 65 Swedish municipalities, were assessed for SUD and their data were available in the research ASI database. About one in four assessments $(n=3731)$ were completed with adults 50 years of age and older at the interview date. This study included those who reported that they had been troubled by alcohol problem at least one day in the past 30 days before their baseline assessment (individuals with harmful alcohol use) $(n=1830)$. Individuals $(n=83)$ with 3 or more class identifying variables missing were excluded from the analysis. Thus, the total study population consisted of 1747 individuals (men: $n=1255$; women: $n=492$ ).

The study was conducted within the project "Studying social services, treatment and other interventions for Alcohol and Narcotics and resulting health outcomes (STANCE)'. The Swedish National Board of Health and Welfare, the Regional Ethical Review Board at Umeå University, and the University of Denver Institutional Review Board (IRB) reviewed and approved the study protocol. All study data were de-identified and the study met IRB exemption criteria.

\section{Measures}

The variables used in the analysis were either ASI items or constructed from ASI items. Response were either client self-reports or interviewer assessments.

\section{Class Membership Indicators}

Eleven indicators, focusing both on lifetime problems (excluding the 30 days before the interview) and recent problems (within the last 30 days) in 6 ASI dimensions, were used to identify subtypes of harmful alcohol use. The employment dimension was excluded because many of its items were not relevant for older adults. Instead, some items from the employment dimension were used in post LCA analysis to examine the generated classes. All of the indicators, except the indicator which measures problem days with physical health, are part of the Swedish ASI "critical items". All indicators were coded to binary, as described below.

(a) Age of onset of regular drinking. Age when the individual started drinking alcohol 3 or more times a week, regardless of amount. The distribution was dichotomized at the median-split - 30 years.

(b) Age of onset of drinking to intoxication level (heavy drinking): (a.) age when the individual started drinking for at least two consecutive days to a level where social, occupational, cognitive or physical functioning are impaired for at least 2 consecutive days or (b.) Age when the individual started drinking 4 (for women) or 5 (for men) standard drinks per day, at least 3 times a week. The distribution was dichotomized at the median-split 35 years.

(c) Recent conflict with family: was based on the ASI question regarding the number of days in the past 30 days the individual had serious interpersonal conflicts with their family. Responses were dichotomized indicating absence or presence of problem days with family relationship.

(d) Lifetime history of adult criminal charges and arrests which are not directly related to alcohol offences: individuals reported the number of times they have been arrested and charged in their lifetime with the following: drug charges; burglary/larceny/shoplifting; violent offences (robbery, assault, homicide/manslaughter); other offences (vandalism/ weapons offence). Responses were dichotomized indicating absence or presence of any of these charges or arrests. 
(e) Life time history of polydrug use: regular use of more than one type of drug, excluding sedatives, for at least 6 months. This indicator is coded from separate reports of use of each drug listed in the ASI (heroin, methadone, barbiturates, other opiates, cocaine, amphetamines, cannabis, hallucinogens, and inhalants).

(f) Life time history of sedative or hypnotic drugs use: regular use of sedatives at least for 6 months.

(g) Recent drug related problems: this indicator item is coded from absence or presence of problem days directly related to drug use in the past 30 days. The problems include craving, physical dependence, disturbing effects of use and unsuccessful efforts to cut down drug use.

(h) Life time history of depression: indicator on absence or presence of depression over extended period of time (at least 2 weeks), unrelated to the individual being under the direct effects of substance use or withdrawal.

(i) Life time history of anxiety: indicator on absence or presence of anxiety over extended period of time (at least 2 weeks), unrelated to the individual being under the direct effects of substance use or withdrawal.

(j) Life time history of trouble controlling violent behavior: this item measures both brief and extended presence of loss of control over violence including episodes of rage, whether the individual was under influence of substance (or withdrawal) or not.

(k) Recent physical health problems: this item is coded from absence or presence of somatic problems which cannot be reversed by abstaining from substance use: as presented in the ASI, "How many days have you experienced medical problems in the past 30 days?"

\section{Demographics}

Demographic variables included age, gender, marital, educational and employment status. In this study, age was categorized into four age groups (50-54, 55-59, 60-64 and $65+$ years old). Education level was categorized as: less than 9 years, above 9 years but below 12 years, 12 years and more than 12 years of education. Finally, the separate "married" and "cohabiting" response categories for marital status were combined to "married/living with partner". Demographic characteristics of the total study sample are shown in Table 1.

\section{Other Variables}

Interviewer Severity Ratings (ISRs): ISRs are seven separate ratings for the seven problem areas and are completed by the clinical social worker after concluding the interview questions in each problem domain. Severity in this

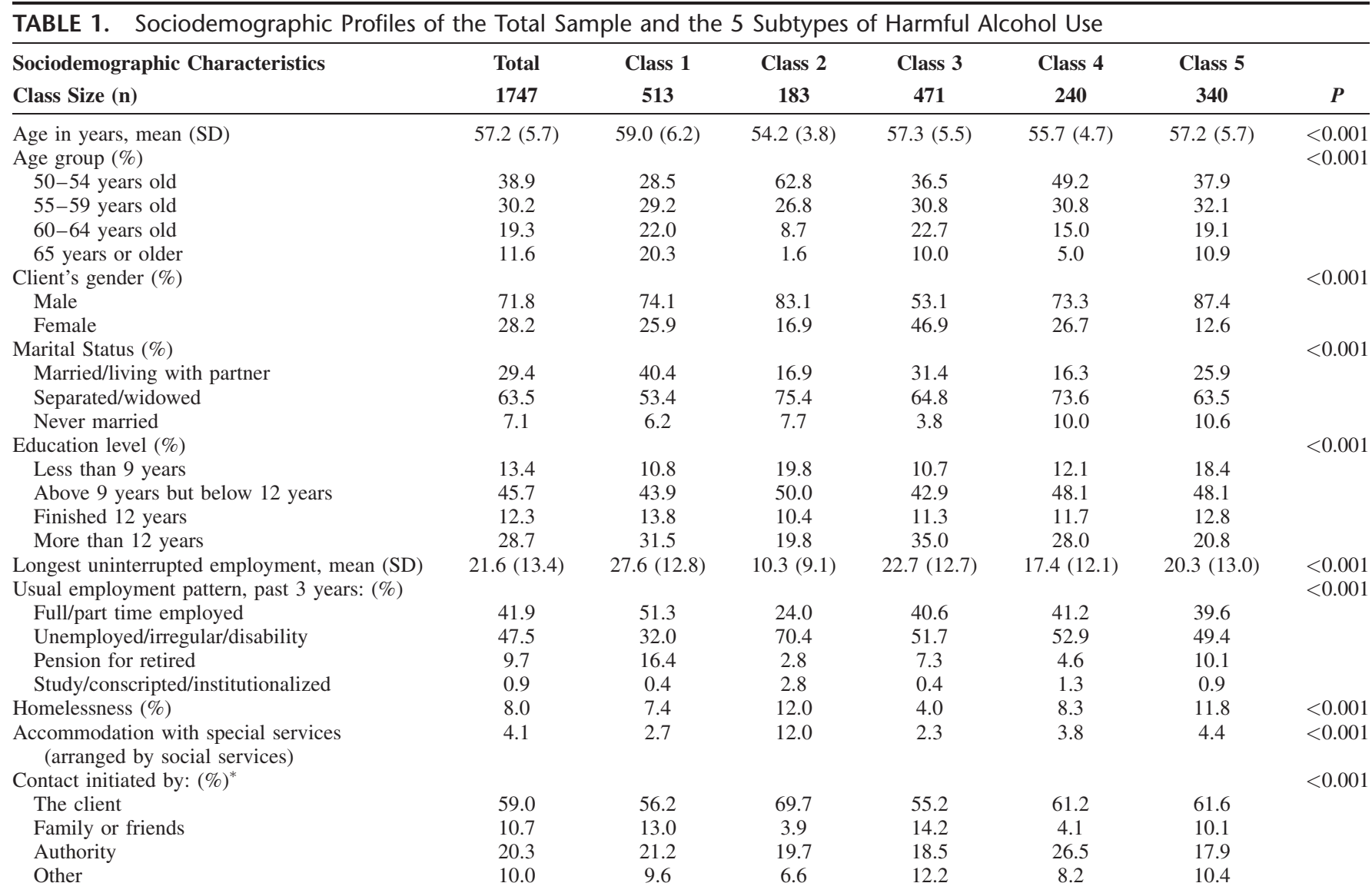

${ }^{*}$ This item was available only for individuals assessed with the 2007 version of Swedish ASI $(\mathrm{N}=1465)$.

Class 1 = Late Onset/ Functional with fewer consequence; Class $2=$ Early onset/ multidimensional problems; Class $3=$ Late Onset with co-occurring Anxiety \& Depression; Class $4=$ Early onset with co-occurring psychiatric problems; Class $5=$ Early onset/ major alcohol problem; SD $=$ Standard deviation. 
rating is defined as "the need for treatment where there currently is none; or for an additional form or type of treatment where the patient is currently receiving some form of treatment". ISRs range between 0 and 9 where $0-1$ ("no real problem, treatment not indicated"), 2 to 3 ("slight problem, treatment probably not necessary"), 4 to 5 ("moderate problem, some treatment indicated"), 6 to 7 ("considerable problem, treatment necessary") and 8 to 9 ("extreme problem, treatment absolutely necessary").

\section{Analysis}

Latent Class Analysis (LCA) identified subtypes of harmful alcohol use based on the 11 class membership indicators. LCA is a person-centered, model-based approach used to identify mutually exclusive subgroups (classes) from the baseline study population based on patterns of responses to a set of observed indicators, where the numbers and structures of subgroups are unknown a priori (Collins and Lanza, 2009; Neely-Barnes, 2010). LCA gives estimates of class prevalence and probabilities of endorsing indicator items conditional to subgroup membership. This procedure means multidimensional classes can be derived where members of one class are most similar to each other, yet most distinct from members of other classes. Parameters in the latent class models were estimated using full information maximum likelihood estimation (FIML) with multiple random start values.

The number of classes of harmful alcohol use was determined after fitting models iteratively until a goodness of fit indices indicated the most parsimonious solution. The Bayesian Information Criterion (BIC), Voung-Lo-MendellRubin (VLMR $P$ value) and the bootstrap likelihood ratio test (BLRT $P$ value) were used to select the best class solution (Nylund et al., 2007) which was then investigated for conceptual meaningfulness. The classification quality of the selected model was examined by evaluating entropy and average class probabilities for most likely class memberships. Furthermore, following the procedure outlined by Masyn (2013), the standardized residuals for each response pattern with modelestimated frequency of $\geq 1.00$ were evaluated. Standardized residuals with large values $(<-3.00$ or $>3.00)$ indicate response patterns that were more poorly fit. For a well-fitting model, less than $1 \%$ of the standardized residuals would be expected by chance to exceed 3.00.

The best LCA solution was validated by the random splitsample cross-validation procedure (Masyn, 2013). The procedure taken in this procedure included 6 steps. Step 1 split the sample randomly into 2 approximately equally sized datasets (the training sample and the validation sample). Step 2 conducted the LCA for the training sample and retained the model parameter estimates for the best LCA solution (K-class model). Step 3 fitted the K-class LCA model to the validation sample by fixing all the parameters to the retained parameters in step $2-$ this model is restricted. Step 4 evaluated the overall absolute fit of the restricted model based on the validation sample-if the fits are acceptable then the model validates well (Collins et al., 1994). In Step 5 all of the parameter constraints were released from the final K-class model from the validation sample in step 4 and refitted to the same sample - this model is the full model. Step 6 takes the restricted model as nested within the full model and additionally evaluates the acceptability of the model by examining the conditional $\mathrm{G}^{2}$ (likelihood-difference statistic) associated with the difference in degrees of freedom $(d f)$ of the 2 models (McCutcheon, 2002). If the $P$ value of the conditional $\mathrm{G}^{2}(d f)$ is not significant at $P=0.05$, then the model validates well and the LCA solution is considered to be stable across the 2 subsamples.

The discriminative validity of the best LCA solution was assessed by comparing the descriptive measures of characteristics not used to define the harmful alcohol use subtypes. Interviewer severity ratings (ISR) across the seven potential problem areas, histories of inpatient and outpatient treatments for alcohol, drug and psychiatric problems, pension for physical disability, history of trauma and other characteristics were compared between classes. Significance tests compared each characteristic between latent classes using ANOVA or Kruskal-Wallis test for continuous variables (depending on the Gaussian distribution) and Pearson $\chi^{2}$ for categorical variables. Multiple pairwise comparisons between the classes used a Bonferroni correction method, and Dunn's test with Bonferroni correction for highly skewed variables. The statistical analyses were conducted by using Mplus version 8.4 (Muthén and Muthén, 1998-2019) and Stata version 15.1 (StataCorp, 2017).

\section{RESULTS}

\section{Five-Class Solution for Harmful Alcohol Use}

LCA was performed iteratively by starting with one class model. Increasing the number of classes beyond 5 did not improve the LCA fit indices. As shown in Table 2, the BIC was the lowest for the five-class solution and the non-significant VLMR and BLRT $P$ values for six-class solution suggested the five-class model was the best solution. An entropy of 0.809 and average class probabilities for most likely class memberships ranging from 0.859 to 0.919 suggested that the

TABLE 2. Fit indices for LCA Models With 1 to 6 Classes

\begin{tabular}{|c|c|c|c|c|c|c|}
\hline & Number of Free Parameters & AIC & BIC & ABIC & VLMR $P$ Value & BLRT $P$ Value \\
\hline 1-class & 11 & 21121.86 & 21181.98 & 21147.04 & - & - \\
\hline 2-class & 23 & 19736.30 & 19862.01 & 19788.94 & 0.000 & 0.000 \\
\hline 3-class & 35 & 19310.28 & 19501.58 & 19390.39 & 0.0049 & 0.000 \\
\hline 4-class & 47 & 18929.88 & 19186.76 & 19037.45 & 0.0023 & 0.000 \\
\hline 5-class & 59 & 18733.09 & 19055.57 & 18868.13 & 0.000 & 0.000 \\
\hline 6-class & 71 & 18676.31 & 19064.37 & 18838.81 & 0.1089 & 0.15 \\
\hline
\end{tabular}

ABIC, adjusted BIC; AIC, Akaike's Information Criterion; BIC, Bayesian Information Criterion; BLRT, bootstrap likelihood ratio test; VLMR, Voung-Lo-Mendell-Rubin. 
chosen solution successfully identified the empirical subtypes of harmful alcohol use among the study population. Further examination of the standardized residuals showed that only four of the observed 787 response patterns $(0.5 \%)$ with modelestimated frequencies above 1.0 had standardized residuals $>3.00$, and all of them $<5.00$ which supported the fit of the five-class solution.

Results from the random split-sample cross-validation procedure supported the five-class model's robustness. The two subsamples, the training $(n=873)$ and the validation $(\mathrm{n}=874)$ subsamples, did not have statistically significant differences in endorsement of the 11 indicator items or demographic characteristics. An iterative LCA of the training sample up to six classes also supported the five-class model with the fit-indices of $\mathrm{BIC}=9,734.85, \mathrm{G}^{2}(1958)=561.92$, $P=1.00$, with size of the five classes being $29.32 \%, 7.79 \%$, $28.75 \%, 15.81 \%$ and $18.33 \%$, and with an entropy of 0.813 . The five-class solution was specified for the validation sample with all the parameters constrained to be equal to those from the model on the training dataset. The obtained fit indices were similar with BIC $=9348.56, \mathrm{G}^{2}(2009)=618.03$, $P=1.00$, with size of the five classes being $29.91 \%$, $9.27 \%, 25.29 \%, 14.3 \%$, and $21.17 \%$, and with an entropy of 0.803 . Finally, the conditional $\mathrm{G}^{2}$ test $(31.76,52 d f)$ between the nested models (five-class fixed-parameter vs free-parameter models) from the validation sample verified the validity of the final model.

The sociodemographic characteristics of the members in the five classes are shown in Table 1. Supplementary Table 1, http://links.lww.com/JAM/A183 presents gender, age-group and education level distribution across the five classes.

Ages of onset of regular and heavy drinking and other variables used to examine the discriminative validity of the model and histories of treatments for alcohol, drug, somatic and psychiatric problems are presented in Table 3. The conditional probabilities of endorsing the indicator items are shown for each class in Figure 1. Those items which were endorsed by less than $30 \%$ (considered as low endorsement) and more than $70 \%$ (considered as high endorsement) of the class members were identified by their location with respect to the reference lines (horizontal dashed lines). Finally, in Figure 2, average ISR for the 5 classes across ASI problem areas are presented. The dashed line at ISR $=4$ specified the thresholds for treatment indication for the referred problem areas.

\section{Description of the 5 Classes}

Class 1 ("Late Onset/ Functional with fewer consequence") accounted for $29.4 \%$ of the total sample and was comprised of $26 \%$ women. Low endorsements on the 11 indicator items characterize Class 1 with late onset of regular drinking (41 years of mean age) and heaving drinking (47 years of mean age) and few adverse consequences. Members of this class had an average age of 59 years and $20 \%$ were 65 years or older. About $40 \%$ were married or living with their partners, a proportion higher than in other classes. Despite being comprised of relatively older individuals, this class reported low number of problem days with loneliness (mean
5.5 days) in the past 30 days, the longest uninterrupted employment (mean 27.5 years) and the lowest proportion of unemployment and disability benefits in the past 3 years $(32 \%)$. More than half of the class members $(51 \%)$ had either fulltime or part-time employment and $45 \%$ had completed 12 years or more education.

Class 2 ("Early Onset/ Prevalent Multi-Dimensional problems") represented $10.48 \%$ of the total sample (women $17 \%)$ and was characterized with early onset of regular (20 years of mean age) and heavy drinking (22 years of mean age), and multiple legal, employment and health problems. Members of this class had a mean age of 54.2 years, had first drunk to intoxication level at a very young age (12.6 years) and drank to that level for a total of 21 years in average. Paternal alcohol use problem was also reported by about $60 \%$ of classmembers. Only $17 \%$ were married or living with partner, $70 \%$ had not completed high school and the longest continuous employment in this subgroup was only 10 years, which was the shortest, compared to the other classes. Additionally, 70\% of the class members reported that they were unemployed or on disability allowances in the past 3 years. Men and women in this class had very high proportion of reported abuse (emotional abuse: $51 \%$ men, $84 \%$ women; physical abuse: $45 \%$ men, $83 \%$ women; sexual abuse: $12 \%$ men, $58 \%$ women). As presented in Figure 1, this class was characterized by concurrent drug, legal and psychiatric problems. Finally, $60 \%$ of the class members had hepatitis B or $\mathrm{C}$ diagnoses.

Class 3 ("Late Onset with co-occurring Anxiety \& Depression"), was characterized with a late onset for regular and heavy drinking 40 years and 45 years old respectively and co-occurring lifetime anxiety and depression. The Class represented of $27.0 \%$ of the total sample and women represented $46 \%$ of the class membership. Members reported an elevated day with loneliness (10.5 days) and elevated levels of recent depression and anxiety, and histories of emotional, sexual and physical abuse. Moreover, $30 \%$ of women in this class reported they were sexual abuse victims, $68 \%$ of women were emotionally abused and $56 \%$ of women had a history of physical abuse.

Class 4 ("Early Onset with co-occurring Psychiatric Problems") accounted for $13.7 \%$ of the total sample and included 27\% women. The class had mean ages of onset for regular and heavy drinking 20 years and 21 years, respectively, elevated proportions of lifetime and current anxiety and depression, high prevalence of lifetime suicidal ideation and attempt, moderate endorsement of criminality indicator and elevated levels of charges for disorderly conducts, lowest proportion of married status. Only $4.1 \%$ of classmembers were referred by family and friends for assessment of SUD.

Class 5 ("Early Onset/ major Alcohol Problem") included $19.5 \%$ of the sample and was predominantly men (87\%) and presented early onsets of regular and heavy drinking (21 years and 23 years old, respectively), and low probabilities of endorsing other indicator items. However, compared to the first class, this class reported more prior treatment episodes for alcohol problems and reported more incidences of delirium tremens, charges for disorderly 


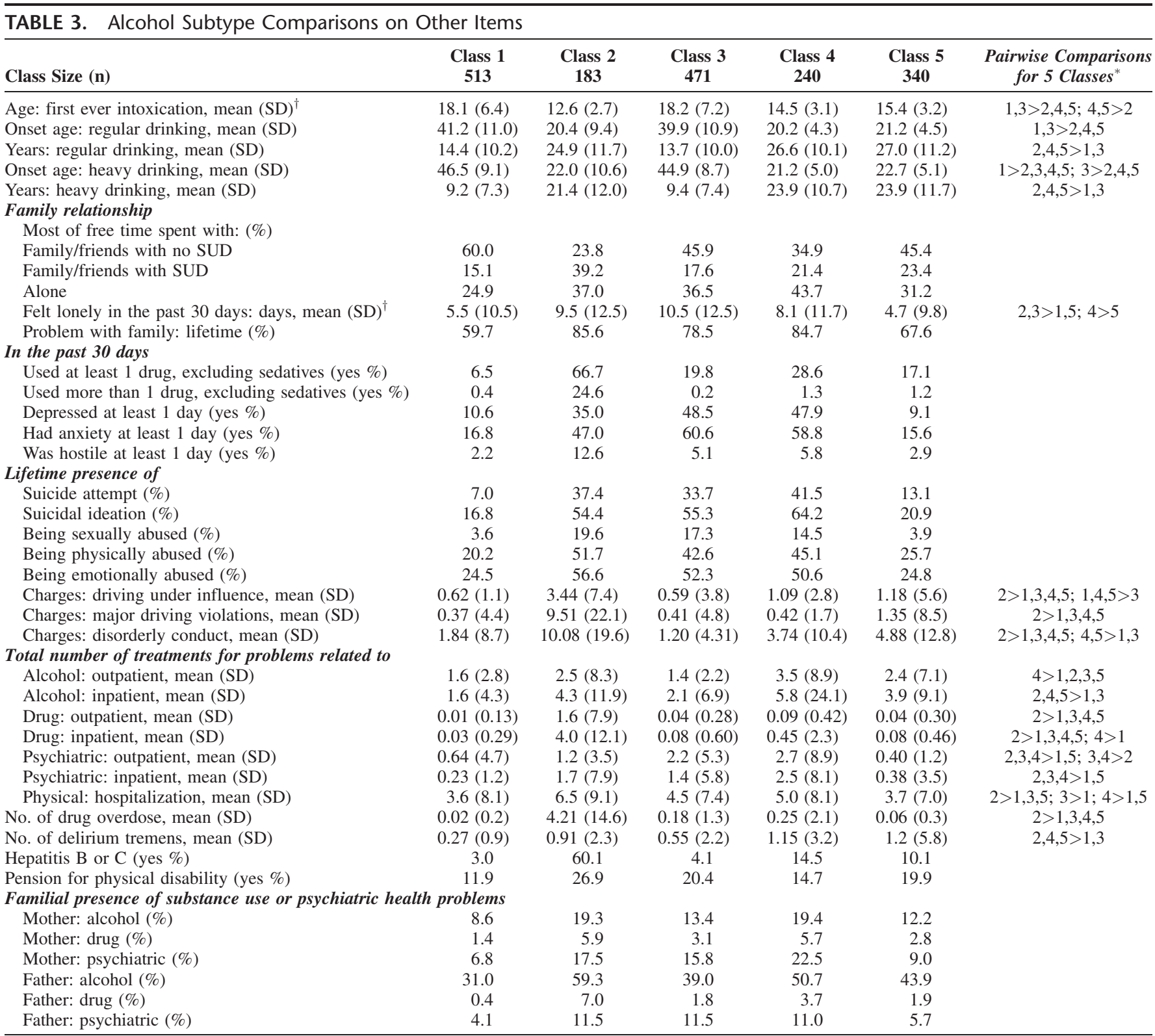

*Pairwise comparisons which were statistically significant at $P$ value $<0.05$ are presented.

these items were available only for individuals assessed with the 2007 version of Swedish ASI $(\mathrm{N}=1465)$ Note. The overall differences for all items across the classes are statistically significant $(P$ value $<0.001)$ except for Mother: drug (maternal drug use) which was significant at $P$ value $=0.007$.

Class $1=$ Late Onset/ Functional with fewer consequence; Class $2=$ Early onset/ multidimensional problems; Class $3=$ Late Onset with co-occurring Anxiety \& Depression; Class $4=$ Early onset with co-occurring psychiatric problems; Class $5=$ Early onset/ major alcohol problem; SD, Standard deviation.

conduct and members were less likely to be married or living with a partner.

\section{Treatment History for Alcohol, Drug and Psychiatric Problems and ISRs Across Classes}

Post-LCA comparisons of outpatient and inpatient treatment histories for alcohol, drug and psychiatric problems examined the concurrent validity of identified typologies of harmful alcohol use and indicated some class-differencesClass 1 reported a few treatment episodes. Class 2 reported multiple treatment episodes for alcohol, drugs other than alcohol and psychiatric problems, Class 3 and 4 reported repeated treatments for psychiatric problems, and Class 4 and 5 had repeated treatments for alcohol problems (see Table 3 ).

The interviewer severity ratings (ISRs) assessing need for treatment were in agreement with severity of problem areas identified by the LCA. The overall Class differences of interviewer severity ratings across the problem areas were significant, except for employment and support $(P=0.072)$ and alcohol problem $(P=0.37)$. 


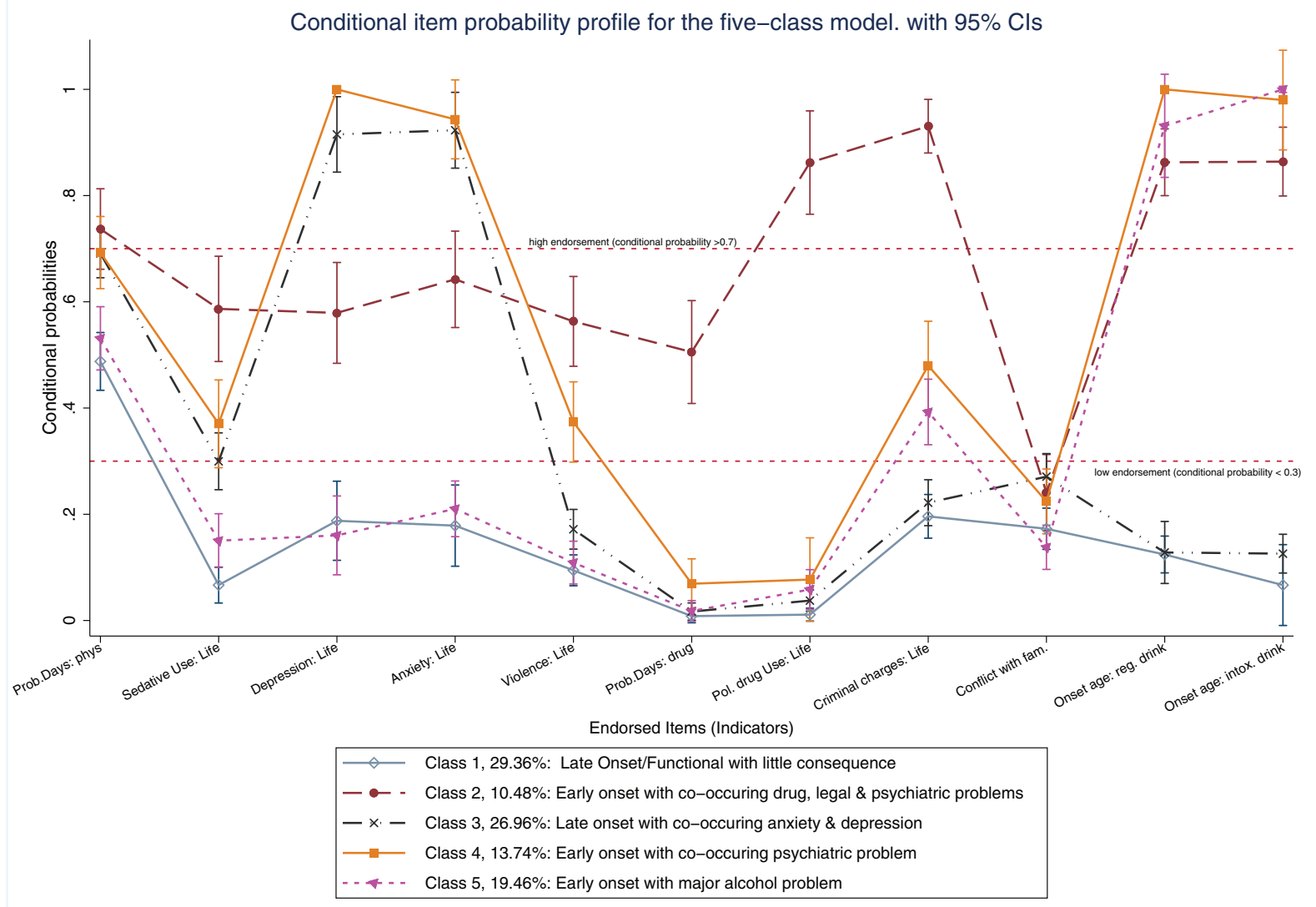

FIGURE 1. Conditional item probability profile for the five-class model.

\section{DISCUSSION}

The analysis identified empirical multidimensional subtypes of harmful alcohol use in adults aged 50 years and older who were assessed for substance use disorders by social workers and reported at least 1 problem day with alcohol in the past 30 days. The study may be the first empirical ASIbased harmful alcohol use typology study focusing on older adults. The LCA analysis on 11 indicators of life domains

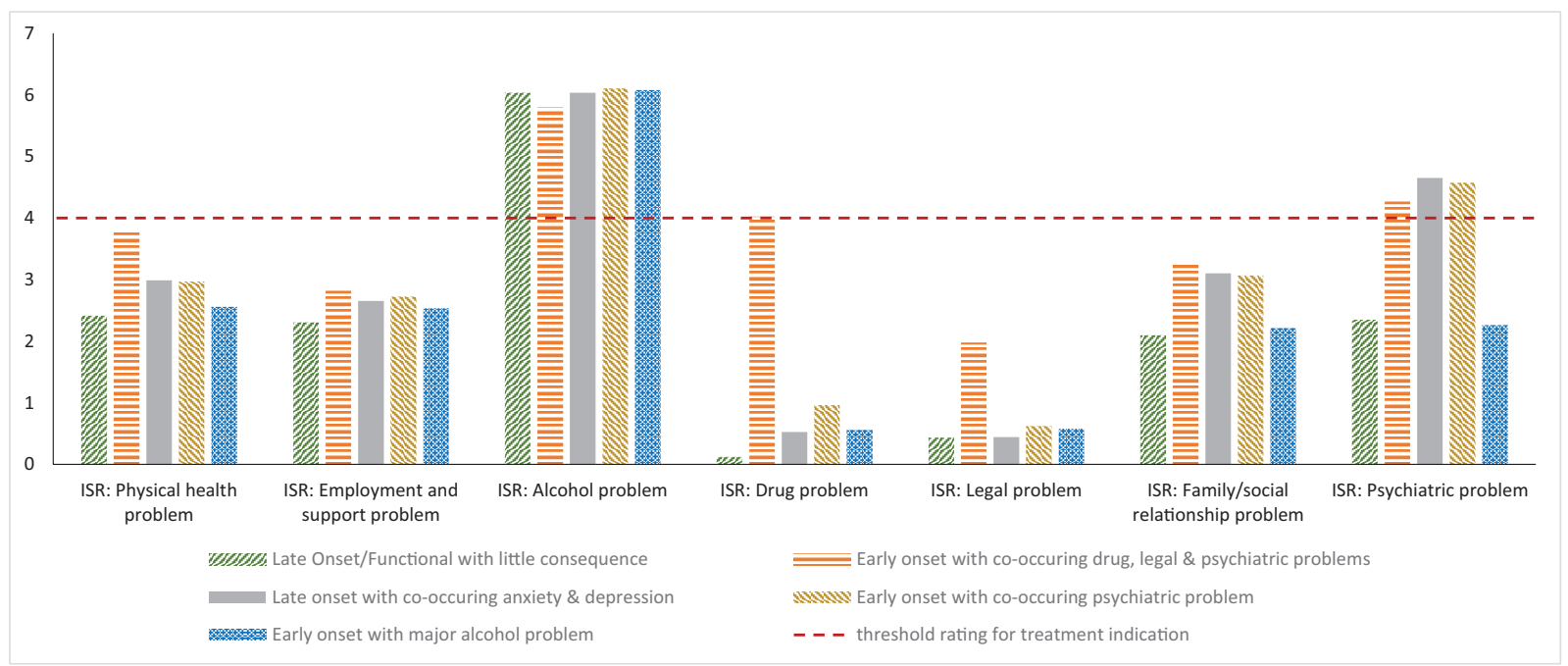

FIGURE 2. Mean Interviewer Severity Ratings for the 5 classes across ASI problem areas. 
commonly affected by substance use disorder identified 5 distinct subtypes, 3 with early onset and 2 with late onset of regular and heavy drinking. The Classes varied in occurrence and severity of use of other substances, psychiatric comorbidities, somatic health and criminality, treatment histories, interviewer severity ratings, and recent presence of psychiatric problems. The Class indicators which were used in the LCA included both lifetime and recent measures of problem dimensions and they belonged to a set of critical questions as used in the Swedish version of the ASI manual.

Our findings support the growing evidence that binary classification of individuals with alcohol use disorders does not sufficiently address the complexity and heterogeneity of the problem (Leggio et al., 2009). Further, it is important to notice that prior samples in alcohol typology studies were composed primarily of younger individuals (Cloninger et al., 1981; Babor et al., 1992b; Epstein et al., 2002; Windle and M. Scheidt, 2004; Moss et al., 2007). Despite this, the different classes identified in our study have similarities to previous results.

The Class 1 subtype, despite comprising older individuals, is marked by fewer consequences in life-domain problem dimensions, and smaller chronicity of alcohol problem. This subtype resembles "type A" from the binary model of Babor et al. (1992b), "type 1" from 3-cluster model of Hauser and Rybakowski (1997), "mild" types from the 4-cluster models of DelBoca and Hesselbrock (1996) and Windle and M. Scheidt (2004), and the "functional class" in the 5-type model of Moss et al. (2007).

Class 2, which is characterized by concurrent drug, legal and psychiatric problems, is similar to "type B" group from Babor's binary classification, the "polydrug" subtype from Windle and Scheidt study and the "High risk/severity" group from Del Boca and Hesselbrock model.

Class 3, a distinct subtype characterized with late onset harmful alcohol use and concurrent psychiatric comorbidity, was not identified in either Babor's or Hauser and Rybakowski's models. Babor's model was binary, and Hauser and Rybakowski's sample was exclusively male. The reanalysis of Babor's data by DelBoca and Hesselbrock (1996), however, suggested two additional subtypes, one of which ("Internalizing" type) was similar to our Class 3 with regard to higher female representation and co-occurring psychiatric problems (depression and anxiety).

Class 4 also had an elevated prevalence of psychiatric problems. Early first intoxication and early-onset of harmful alcohol use, higher criminality and higher lifetime prevalence of suicide ideation and attempts, higher episodes of rage and other violent behaviors characterize Class 4 compared to Class 3. Other studies (DelBoca and Hesselbrock, 1996; Windle and M. Scheidt, 2004) produced similar subtypes to Class 4. Their results differ from ours with respect to their clusters reporting lower prevalence of depression and anxiety. Hauser and Rybakowski (1997), however, reported "type 3" which was characterized by early onset harmful alcohol use and concurrent psychiatric and somatic health problems. Their "type 3" subgroup reported higher prevalence of other drugs dependency compared to the Class 4 subgroup. In our study, Class 4 members reported low proportion of lifetime sedative use and very low prevalence of lifetime polydrug use and drug problem days.

The co-occurrence of SUD and psychiatric disorders is widely recognized. Ross and Peselow (2012) and Kelly and Daley (2013) suggest that approximately $50 \%$ of those with a mental health disorder have at some time in their life had a SUD and vice versa. Even if the present study does not address temporality of causation between harmful alcohol use and psychiatric comorbidity, high prevalence of depression, anxiety and lonely days is observed along with history of abuse in Class 2, Class 3, and Class 4 members supporting findings from previous researches that psychiatric problems and prior trauma are associated with harmful alcohol use.

The high psychiatric comorbidity in the Class 4 subgroup, unlike the Class 3 subtype, coexisted with higher prevalence of criminality, disruptive behavior and hostility, and higher frequency of delirium tremens. The results from our study suggest that the individuals in this class may be socially isolated in addition to co-occurring psychiatric problems and chronic heavy drinking. Different study designs with additional measurement instruments are required to study causality links between the harmful alcohol use and mental health disorders observed within the Class 3 and Class 4 subtypes.

None of the prior alcohol typology studies report subtypes similar to Class 5. This most likely is due to the difference in ages of the different study samples. A previous Swedish ASI register based study with a national sample of 12,833 individuals assessed for SUD had reported an "alcohol profile" (Lundgren et al., 2014) with lower interviewer severity rating for alcohol problem. This suggests that severity of alcohol problem among the Class 5 members might worsen as they age. Additionally, while Lundgren et al. (2014) reported 3 clusters (narcotics, alcohol and alcohol with psychiatric comorbidity), our study was able to identify 5 distinct classes, all with varying severity of ASI problem areas.

It is important to note that the study by Lundgren et al. (2014) used the interviewer severity ratings for categorization and employed cluster analysis to identify substance use disorder profiles and did not focus on alcohol. The study population in that study was mainly young adults and included all individuals who were assessed with the ASI. The present study, in contrast, used LCA with multiple objective indicators across problem areas, fit indices selected the best class solution and its study population was composed of adults aged 50 years and older who have reported at least one alcohol problem day in the last 30 days.

The present study provides evidence that older adults with harmful alcohol are not a homogeneous group. A portion of the older population have multidimensional problems with intervention needs for concurrent drug use and psychiatric morbidity. Prior investigations examined differences among older adults with alcohol dependency; nevertheless, their use of age of onset of alcohol dependency as the only classification criterion had resulted in a conclusion that adults with late onset of alcohol dependency have similar health profile (Wetterling et al., 2003; van den Berg et al., 2014; Schückher et al., 2017; Van Montfoort-De Rave et al., 2017). Using multiple indicators, we documented a class with late harmful 
alcohol use onset (Class 3) who reported high psychiatric comorbidity and should probably not be defined as healthy and another class with fewer consequences in life-domain problem dimensions (Class 1).

This study has strengths and limitations. The use of multiple indicators and application of LCA for classification produced distinct subtypes of harmful alcohol use among older adults. SUD assessments in Sweden are conducted by trained clinical social workers certified to use ASI, which assures assessment quality. This can be seen in the alignment of the interviewer severity ratings with the classes obtained from a posteriori analysis. The findings of this study, however, are based on a single assessment tool which may introduce methodological bias because the ASI does not capture other important domains related to alcohol use disorder, such as personality and drinking motives. The ASI is a validated tool that measures multidimensional aspects of SUD, and is widely used in Sweden and many other countries in a range of populations and settings.

The cross-sectional design of this study does not allow us to examine the stability of the identified classes over time. Individuals may manifest varying severity of harmful alcohol use and move in and out of harmful alcohol use classes over time. Additional data will be available in the near future, and we plan longitudinal studies to monitor transitions from one class to another and differences in health outcomes and classresponse to interventions. Another limitation regarding the generalizability of the findings is that the study utilizes ASI self-report and standardized assessment data from treatment seekers. This may introduce Berkson's bias, a type of selection bias which arises from sampling that is not conducted in the general population.

As in many substance use studies, this study is prone to reporting biases. Nonetheless, reliability of the ASI has been studied and verified. It is recognized, moreover, that because of age-related physiological and cognitive deterioration, some older adults are more vulnerable to the effects of substance use, pharmacodynamic and pharmacokinetic changes, harmful drug-drug and drug-disease interactions and co-morbid chronic diseases. Defining "intoxication" as drinking 4 (for women) or 5 (for men) standard drinks per day, as in the ASI, does not consider increased age-related sensitivity to alcohol in some older adults.

In conclusion, the design of effective intervention programs starts from identification of groups with specific needs and characteristics. Our study suggests that significant portion of older adults with harmful alcohol use present additional needs of intervention for concurrent illicit drug use and comorbid psychiatric disorders. Our results also suggest that adults with late onset of alcohol dependency do not necessarily have better health profile when compared to adults with early onset of alcohol dependency.

The findings from our study may inform treatment/ intervention providers, when considering intervention options. Older adults with comparable severity of alcohol problem do not necessarily have similar needs for treatment and other clinical and demographic differences should be considered if older adults with harmful alcohol use are to benefit from services and treatments.

\section{ACKNOWLEDGMENTS}

We acknowledge and have great appreciation for all the work of Professor Bengt-Ake Armelius, Professor Kerstin Armelius and Dr. Siv Nystrom. These individuals were the initial implementers of the Addiction Severity Index assessment instrument in Sweden. We also want to thank all municipalities who permitted us to use their registry data.

\section{REFERENCES}

Akerlind I, Hornquist JO. Loneliness and alcohol abuse: a review of evidences of an interplay. Soc Sci Med 1992;34:405-414.

Armelius B-A, Nyström S, Engström C, et al. Referensmaterial för bättre använding av ASI intervjun [Reference material for better use of the ASI interview]. The Institute for the Development of Methodology in Social Work. Socialstyrelsen (National Board of Health and Welfare). Stockholm, Sweden; 2009.

Arndt S, Clayton R, Schultz SK. Trends in substance abuse treatment 19982008: increasing older adult first-time admissions for illicit drugs. Am J Geriatr Psychiatry 2011;19:704-711.

Babor TF, Dolinsky ZS, Meyer RE, et al. Types of alcoholics - concurrent and predictive-validity of some common classification schemes. Brit J Addict 1992;87:1415-1431.

Babor TF, Hofmann M, Delboca FK, et al. Types of alcoholics. 1. Evidence for an empirically derived typology based on indicators of vulnerability and severity. Arch Gen Psychiat 1992;49:599-608.

Badrakalimuthu VR, Rumball D, Wagle A. Drug misuse in older people: old problems and new challenges. Adv Psychiatr Treat 2010;16:421-429.

Beynon CM. Drug use and ageing: older people do take drugs! Age Ageing 2009;38:8-10.

Bohman M, Sigvardsson S, Cloninger CR. Maternal inheritance of alcohol abuse. Cross-fostering analysis of adopted women. Arch Gen Psychiatry 1981;38:965-969.

CAN. Drug-related deaths in Sweden- Estimations of trends, effects of changes in recording practices and studies of drug patterns. Stockholm: Centralförbundet för alkohol- och narkotikaupplysning. In: Report 158, 2016.

Carew AM, Comiskey C. Treatment for opioid use and outcomes in older adults: a systematic literature review. Drug Alcohol Depend 2018;182: 48-57.

Cloninger CR, Bohman M, Sigvardsson S. Inheritance of alcohol abuse. Cross-fostering analysis of adopted men. Arch Gen Psychiatry 1981;38: $861-868$.

Collins LM, Graham JW, Long JD, Hansen WB. Crossvalidation of latent class models of early substance use onset. Multivariate Behav Res 1994;29:165-183.

Collins LM, Lanza ST. Latent Class and Latent Transition Analysis | Wiley Series in Probability and Statistics. 2009.

DelBoca FK, Hesselbrock MN. Gender and alcoholic subtypes. Alcohol Health Res W 1996;20:56-62.

Epstein EE, Labouvie E, McCrady BS, et al. A multi-site study of alcohol subtypes: classification and overlap of unidimensional and multi-dimensional typologies. Addiction 2002;97:1041-1053.

Flint A, Merali Z, Vaccarino FE. Substance use in Canada: improving quality of life: substance use and aging. Canadian Centre on Substance Use and Addiction; 2018.

Graham K, Zeidman A, Flowers MC, et al. A typology of elderly persons with alcohol problems. Alcohol Treat $Q$ 1993;9:79-95.

Han B, Gfroerer JC, Colliver JD, Penne MA. Substance use disorder among older adults in the United States in 2020. Addiction 2009;104: 88-96.

Hauser J, Rybakowski J. Three clusters of male alcoholics. Drug Alcohol Depend 1997;48:243-250.

Hesselbrock VM, Hesselbrock MN. Are there empirically supported and clinically useful subtypes of alcohol dependence? Addiction 2006;101(Suppl 1):97-103.

Jellinek EM. Alcoholism, a genus and some of its species. Can Med Assoc J 1960;83:1341-1345.

Kelly TM, Daley DC. Integrated treatment of substance use and psychiatric disorders. Soc Work Public Health 2013;28:388-406. 
Kim S, Spilman SL, Liao DH, et al. Social networks and alcohol use among older adults: a comparison with middle-aged adults. Aging Ment Health 2018;22:550-557.

Kuerbis A, Sacco P. The impact of retirement on the drinking patterns of older adults: a review. Addict Behav 2012;37:587-595.

Kuerbis A, Sacco P, Blazer DG, et al. Substance abuse among older adults. Clin Geriatr Med 2014;30:629-654.

Kuerbis A, Treloar Padovano H, Shao S, et al. Comparing daily drivers of problem drinking among older and younger adults: an electronic daily diary study using smartphones. Drug Alcohol Depend 2018;183:240-246.

Leggio L, Kenna GA, Fenton M, et al. Typologies of alcohol dependence. from jellinek to genetics and beyond. Neuropsychol Rev 2009;19:115-129.

Lichtenberg PA. Introduction. In: Lichtenberg PA, editor. Handbook of Assessment in Clinical Gerontology (Second Edition). San Diego: Academic Press; 2010. xi-xvii.

Lundgren L, Krull I. Screening, assessment, and treatment of substance use disorders: evidence-based practices, community and organizational setting in the era of integrated care. New York: Oxford University Press; 2018.

Lundgren L, Wilkey C, Chassler D, et al. Integrating addiction and mental health treatment within a national addiction treatment system: Using multiple statistical methods to analyze client and interviewer assessment of co-occurring mental health problems. Nord Stud Alcohol Dr 2014;31:59-79.

Makela K. Studies of the reliability and validity of the Addiction Severity Index. Addiction 2004;99:398-410. discussion 411-8.

Masyn KE. 25 latent class analysis and finite mixture modeling. In: The Oxford Handbook of Quantitative Methods. Oxford: Oxford University Press, 2013: 551.

McCutcheon AL. Basic concepts and procedures in single- and multiplegroup latent class analysis. In: Hagenaars JA, McCutcheon AL, editors. Applied Latent Class Analysis. Cambridge: Cambridge University Press; 200256-86.

McLellan AT, Kushner H, Metzger D, et al. The fifth edition of the Addiction Severity Index. J Subst Abuse Treat 1992;9:199-213.

Moos RH, Schutte KK, Brennan PL, et al. Older adults' alcohol consumption and late-life drinking problems: a 20-year perspective. Addiction 2009; 104:1293-1302.

Morey LC, Skinner HA, Blashfield RK. A typology of alcohol abusers: correlates and implications. J Abnorm Psychol 1984;93:408-417.

Moss HB, Chen CM, Yi H-y. Subtypes of alcohol dependence in a nationally representative sample. Drug Alcohol Depend 2007;91:149-158.

Muthén LK, Muthén BO. Mplus User's Guide. Eighth ed. Los Angeles, CA: Muthén \& Muthén; 1998-2019.

Neely-Barnes S. Latent Class Models in Social Work. Soc Work Res 2010;34:114-121.
Nylund KL, Asparouhov T, Muthén BO. Deciding on the number of classes in latent class analysis and growth mixture modeling: a Monte Carlo simulation study. Struct Equ Model Multidiscip J 2007;14: 535-569.

Nystrom S, Andren A, Zingmark D, et al. The reliability of the Swedish version of the Addiction Severity Index (ASI). J Subst Use 2010;15: 330-339.

Nyström S, Zingmark D, Jäderland A. ASI-manualen: anvisningar till ASI Grund och ASI Uppföljning [ASI-manual: Instructions for ASI Basic and ASI Follow-up]. In: Socialstyrelsen ed. Socialstyrelsen, 2009.

Perreira KM, Sloan FA. Life events and alcohol consumption among mature adults: a longitudinal analysis. J Stud Alcohol 2001;62:501-508.

Public Health Agency of Sweden. Hälsa på lika villkor. Alkoholvanor, nationella resultat tidsserier 2016 [Health on equal terms. Alcohol drinking habits. National public health survey]. Folkhälsomyndigheten 2016.

Robins E, Guze SB. Establishment of diagnostic validity in psychiatric illness: its application to schizophrenia. Am J Psychiatry 1970;126:983-987.

Rosin AJ, Glatt MM. Alcohol excess in the elderly. $Q$ J Stud Alcohol 1971;32:53-59.

Ross S, Peselow E. Co-occurring psychotic and addictive disorders: neurobiology and diagnosis. Clin Neuropharmacol 2012;35:235-243.

Royal College of Psychiatrists. Our Invisible Addicts (College Report CR211) Royal College of Psychiatrists 2018.

Schückher F, Sellin T, Berglund K, et al. The importance of age at onset of excessive alcohol use with regard to psychiatric symptoms and personality characteristics. Alcohol Treat $Q$ 2017;35:328-343.

Sorocco KH, Ferrell SW. Alcohol use among older adults. J Gen Psychol 2006;133:453-467.

StataCorp. Stata Statistical Software: Release 15. College Station, TX: StataCorp LLC; 2017.

Statistics Sweden. The future population of Sweden 2018-2070. Demographic reports. 2018; 1.

The National Board of Health and Welfare. Vård Och Omsorg Om Äldre [Health and Social Care for the Elderly: Progress report 2018]. In: Lägesrapport, 2018.

van den Berg JF, Hermes JSJ, van den Brink W, et al. Physical and mental health and social functioning in older alcohol-dependent inpatients: the role of age of onset. Eur Addict Res 2014;20:226-232.

Van Montfoort-De Rave KFG, De Weert-Van Oene GH, Beurmanjer H, et al. Late-onset alcohol dependence: patient-reported problems. Addict Res Theory 2017;25:139-145.

Wetterling T, Veltrup C, John U, et al. Late onset alcoholism. Eur Psychiatry 2003;18:112-118.

Windle M, M. Scheidt D. Alcoholic subtypes: are two sufficient? Addiction 2004;99:1508-1519. 